Presented at Orb1t Correction and Analysis Workshop, BlNL, Upton, New York, December 1-3, 1993.

\title{
Eigenvector method for optimized orbit correction*
}

\author{
Aharon Friedman and Eva Bozoki \\ National Synchrotron Light Source, \\ Brookhaven National Laboratory, \\ Upton, NY 11973
}

\begin{abstract}
The task of the orbit correction is to find the kick vector $\Theta$ for a given measured orbit vector $\ddot{X}$. We are presenting a method, in which the kick vector is expressed as linear combination of the eigenvectors. An additional advantage of this method is that it yields the smallest possible kick vector to correct the orbit. We will illustrate the application of the method to the NSLS Xray and UV storage rings and the resulting measurements. It will be evident, that the accuracy of this method allows the combination of the global orbit correction and local optimization of the orbit for beam lines and insertion devices.

The eigenvector decomposition can also be used for optimizing kick vectors, taking advantage of the fact that eigenvectors with corresponding small eigenvalues generate negligible orbit changes. Thus, one can reduce a kick vector calculated by any other correction method and still stay within the tolerance for orbit correction.

The response matrix $A$ is defined by the equation $\vec{X}=A \vec{\Theta}$, where $\vec{\Theta}$ is the kick vector and $\vec{X}$ is the resulting orbit vector. Since $A$ is not necessarily a symmetric or even a square matrix we symmetrize it by using $A^{T} A$. Then we find the eigenvalues and eigenvectors of this $A^{T} A$ matrix. The physical interpretation of the eigenvectors for circular machines is discussed.
\end{abstract}

\section{Introduction}

As it will be discussed later in this section, orbit correction is a relevant issue in any storage ring whether it is an electron synchrotron radiation facility or a high energy machine used for elementary particle or nuclear physics research. It is also important in other circular accelerators, such as booster rings and damping rings. Throughout this paper we will, thus, use the term "circular machine" to signify any circular accelerator (electron or otherwise).

\subsection{The need for accurate orbit correction and stability}

In circular machines, the beam orbit is usually very important to the output of the machine. A good example is synchrotron radiation facilities where the source point and direction of the photon beams depend on the beam position and angle. Since the beam lines are quite long $(10-20 \mathrm{~m})$, any small change in those quantities will yield an unwanted large change in the photon beam position and/or angle at the experiment. In addition, the lifetime of the beam in the machine, the maximum current, and sometimes the ability of the machine to store a beam at all depends on an accurate beam orbit. Not less important is the stability of the orbit (i.e. the time dependent changes in the orbit). An unstable orbit can increase the dynamic aperture of the beam. Thus, reducing lifetime. In a synchrotron facility, an unstable orbit also reduces the effective brightness of the photon source.

*Work performed under the auspices of the U.S. Dept. of Energy under contract no. DE-AC02-76CH00016.

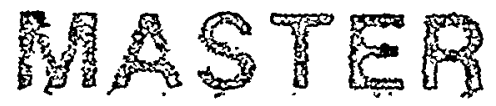


Because the orbit in circular machines is closed, any local change in beam position results in a global change of the orbit. Thus, a misplaced quadruple, an error in a dipole field, a small motion in beam lines, an outside source electromagnetic interference with the beam, all these generate global orbit changes that need to be corrected. In addition, during the operation of the machine some global changes take place, such as a change in circumference due to thermal change, these changes also affect the orbit globally. It is therefore important to implement orbit correction algorithms in the operation procedure of any circular machines.

\subsection{Overview of orbit correction methods}

Most orbit correction methods used in circular machines [1-9] are variations on three 'themes': the harmonic and the least-square algorithms and the superimposition of local bumps. There are also some extra 'twists' which modify these methods [10-12]. In the harmonic methods, the harmonic content of the orbits are calculated and then the corrector strengths are obtained, assuming that the harmonic composition of the dipole field 'errors' reflects that of the orbit. In the least-square methods, the rms orbit all around the machine is minimized. The local bumps method implies that local orbit corrections are calculated all around the ring and then superimposed on each other.

The harmonic and least-square methods complement each other. The harmonic methods are very sensitive to the positioning of the correctors and monitors. They yield good results only when those elements are close to being $\pi / 2$ phase apart. They use many correctors around the machine and the individual kicks are relatively small. The sources of inaccuracies in this method are that (i) the harmonic content of the orbit is calculated from measurements at a finite number of locations, (ii) correcting for the most important few - usually lower - harmonics introduces higher harmonics into the corrected orbit and (iii) some orbits contains high harmonics, which is not easy to correct.

The least-square methods yield very good results in finding and compensating for a small number of dipole 'errors' in the ring. They use a few correctors only but the kicks tend to be much larger then in case of the harmonic methods. Also, when the number of correctors is increased, the correctors start to 'fight' each other, resulting in very large kicks.

The most commonly used least-square algorithm, Micado [2] is based on the Householder transformation and pivoting. When asked to use $\mathrm{N}$ correctors out of the available total of $N_{c}$, the algorithm is choosing the 'most effective' $N$ correctors. Compared with a 'brute force' method, which tries all possible $\left(\begin{array}{c}N_{c} \\ N\end{array}\right)$ combination and accepts the one resulting in the absolute minimum of the residual rms orbit, Micado will result in slightly smaller kicks. As a consequence, sometimes, Micado will point to an equivalent 'most effective' corrector instead of the actual error location in the machine. In addition, the redundancy of correctors in the machine causes the matrix to be not well behaved. Thus, reducing the accuracy of the solution.

\section{The Response Matrix and its eigen states}

Usually in a circular machine, the beam position is monitored by a set of pickup electrodes (PUE), distributed around the machine and orbit correction is performed using a set of distributed dipoles (correctors). The relationship between a change in the strength of a corrector and the corresponding change of the beam position at the PUEs, is expressed by the response matrix. In this section we define and analyze the response matrix.

\subsection{Definition of the Response Matrix}

The orbit change due to a change in the corrector strengths (orbit kick) can be expressed as [13]:

$$
\vec{X}=A \vec{\Theta}
$$




\section{DISCLAIMER}

This report was prepared as an account of work sponsored by an agency of the United States Government. Neither the United States Government nor any agency thereof, nor any of their employees, makes any warranty, express or implied, or assumes any legal liability or responsibility for the accuracy, completeness, or usefulness of any information, apparatus, product, or process disclosed, or represents that its use would not infringe privately owned rights. Reference herein to any specific commercial product, process, or service by trade name, trademark, manufacturer, or otherwise does not necessarily constitute or imply its endorsement, recommendation, or favoring by the United States Government or any agency thereof. The views and opinions of authors expressed herein do not necessarily state or reflect those of the United States Government or any agency thereof. 


\section{DISCLAIMER}

Portions of this document may be illegible in electronic image products. Images are produced from the best available original document. 
where $\vec{\Theta}=\left[\Theta_{j}\right] ; 1 \leq j \leq N_{c}$ is the kick vector, $\vec{X}=\left[X_{i}\right] ; 1 \leq i \leq N_{m}$ is the orbit vector and $A=\left[A_{i, j}\right]$ is the response matrix. The element $A_{i, j}$ of the response matrix is the orbit change on the $\mathrm{i}$-th orbit monitor due to a unit kick from the j-th corrector. In this paper, unless otherwise specified, the beam position and the orbit kick are expressed in $\mathrm{mm}$ and Kdigit ${ }^{1}$ respectively.

\subsection{Eigen solutions of the Response Matrix}

In general, the number of correctors and monitors are different, consequently the response matrix $A$ is rectangular and Eq. (1) is over or under constrained. In order to solve Eq. (1), we left-multiply it by $A^{T}$, the transpose of the response matrix $A$ :

$$
A^{T} \vec{X}=A^{T} A \vec{\Theta}
$$

The solution of Eq. (2) is the least-square approximation of a solution of Eq. (1) (see Ref. [14]). The matrix $A^{T} A$ is symmetric and non negative Ref. [1]. The eigen solutions of Eq. (2) are found by diagonalyzing the matrix $A^{T} A$. This results in the eigenvalues $\left[\lambda_{j}\right]$ and the corresponding eigenvectors ${ }^{2}$ $\left[\hat{\theta}_{j}\right]$. An eigenvector $\hat{\theta}_{j}$ represents a set of corrector values. The orbit change corresponding to the $j$-th eigenvector is:

$$
\vec{x}_{j}=A \hat{\theta}_{j}
$$

It can be shown that

$$
\left(\vec{x}_{j} \cdot \vec{x}_{j}\right)=\lambda_{j},
$$

and that $\vec{x}_{i}$ is an eigenvector of the matrix $A A^{T}$. The physical meaning of the eigenvalues becomes clear from Eq. (4). An eigenvalue $\lambda_{j}$ is a quantitative measure of the orbit response (of the machine) to the $j$-th eigenvector. A small eigenvalue corresponds to a small $\vec{x}_{j}$ orbit change $\left(\left(x_{j}\right)_{r m s}^{2}=\right.$ $\left.\left|\vec{x}_{j}\right|^{2} / N_{m}=\lambda_{j} / N_{m}\right)$. This property of the eigen solutions will be used later in Sec. 4 for reducing the corrector strengths without significantly changing the resulting orbit.

\section{The representation of a circular machine}

In order to give physical interpretation to the response matrix and its eigen solution, one has to look at the dynamics of the beam orbit in a circular machine, and use it to understand the meaning and nature of its eigen solutions. In this section we lay out the dynamics that lead to the response matrix and show the physical meaning of its eigen solution in several cases.

\subsection{Courant-Snyder equation}

The equation describing the beam position in a circular machine is [15].

$$
\frac{d^{2} x}{d s^{2}}+K(s) x=f(s)
$$

where $x$ is the transverse displacement, $s$ is the distance along the equilibrium orbit (measured from some reference point) $K$ is the focusing function and $f$ is the restoring force due to any focusing 'error' (e.g. correctors, errors, noise, etc). For a given set of discrete PUEs and correctors, the solution to Eq. (5) is:

$$
\frac{x_{i}}{\sqrt{\beta_{i}}}=\frac{1}{2 \sin \pi \nu} \sum_{j=1}^{N_{c}} \theta_{j} \sqrt{\beta_{j}} \cos \nu\left(\left|\phi_{i}^{\prime}-\phi_{j}\right|-\pi\right),
$$

\footnotetext{
${ }^{2}$ digits - Digitized voltage of the computer controlled corrector power supply.

${ }^{2}$ The symbol $\hat{v}$ represents that $\vec{v}$ is a unit vector (i.e. $v^{2}=1$ ).
} 
where $\beta_{i}$ is the $\beta$ function value at the PUE in which $x_{i}$ is observed, $\phi_{i}^{\prime}$ is the phase location of that PUE, $\theta_{j}$ is the angular kick introduced to the beam by the corrector located at phase $\phi_{j}, \beta_{j}$ is the $\beta$ function value at $\phi_{j}, \nu$ is the tune (number of betatron oscillations) of the machine, and $N_{c}$ is the total number of correctors.

It is easy to see from Eq. (1) and Eq. (6) that the elements of the response matrix $A$ are:

$$
A_{i j}=\frac{\sqrt{\beta_{i} \beta_{j}}}{2 \sin \pi \nu} \cos \nu\left(\left|\phi_{i}^{\prime}-\phi_{j}\right|-\pi\right) .
$$

Note that in most practical cases, the numerical value of the matrix element $A_{i j}$ cannot be theoretically evaluated to a satisfactory accuracy since the values of $\beta_{i}$ and $\beta_{j}$ are not known well enough. It was actually suggested [16] to use the measured value of the response matrix elements $\left[A_{i j}\right]$ in order to estimate the values of the $\beta$ function.

\subsection{Equidistant correctors and monitors}

Consider a ring in which there are equal number of PUEs and correctors $\left(N_{m}=N_{c}=N\right)$ and they are positioned at equal intervals around the ring. Furthermore, for the sake of simplicity, we assume that the $\beta$ function has the same value for any PUE $\left(\beta_{i}=\beta_{M}\right)$, and that it has the same value for any corrector $\left(\beta_{j}=\beta_{C}\right)^{3}$ Clearly the response matrix in such a case is cyclic since the symmetry is such that the point $i=0$ can be chosen arbitrarily to be any PUE. It is indeed, easy to prove, by way of mathematical induction that Eq. (7) yields a cyclic matrix whose first line is

$$
A_{1 j}=\frac{\sqrt{\beta_{m} \beta_{c}}}{2 \sin \pi \nu} \cos \nu\left[\frac{2 \pi}{N}\left(j-\frac{1}{2}\right)-\pi\right] \text {. }
$$

The eigenvectors of a cyclic matrix are found in Ref. 19. Substituting them for the matrix in Eq. (8), results in

$$
\lambda_{j}=\frac{1}{2} \exp \left[i \pi j\left(1-\frac{1}{N}\right)\right] \frac{\sin \pi(j+\nu)}{\sin \frac{\pi}{N}(j+\nu)}+\frac{1}{2} \exp \left[-i \pi j\left(1-\frac{1}{N}\right)\right] \frac{\sin \pi(j-\nu)}{\sin \frac{\pi}{N}(j-\nu)} .
$$

As it is expected [17], the orbit response to the $j$-th harmonic $\left(\lambda_{j}\right)$ gets larger as $j$ gets closer to the tune $\nu$.

\subsection{Perfectly super-symmetric ring}

In a super symmetric ring, there are $N_{s}$ super symmetric sections. The sections are identical in $\beta$ function and in the placing of trims and PUEs. To simplify the algebra, we express Eq. (6) in a complex format.

$$
\frac{x_{i}}{\sqrt{\beta_{i}}}=\frac{1}{2 \sin \pi \nu} \sum_{j=1}^{N_{c}} \theta_{j} \sqrt{\beta_{j}} \exp i \nu\left(\left|\phi_{i}^{\prime}-\phi_{j}\right|-\pi\right) .
$$

The real orbit can be obtained by taking the real part of $x_{i}$. The response matrix can now be written as a matrix of sub matrices.

$$
A=\left[\begin{array}{cccc}
R & e^{i \nu 2 \pi / N} R & \ldots & e^{i \nu\left(N_{*}-1\right) \pi / N_{\bullet}} R \\
e^{i \nu\left(N_{*}-1\right) \pi / N_{*}} R & R & \ldots & e^{i \nu\left(N_{*}-2\right) \pi / N_{\bullet}} R \\
\vdots & \vdots & \ddots & \vdots \\
e^{i \nu 2 \pi / N_{\bullet}} R & e^{i \nu 4 \pi / N_{\bullet}} R & \ldots & R
\end{array}\right]
$$

\footnotetext{
${ }^{3}$ This assumption does not limit the generality of the solution, since the positions and angles can be normalized by $x_{i}^{N}=x_{\imath} / \sqrt{\beta_{i}}$ and $\theta_{j}^{N}=\theta, \sqrt{\beta_{3}}$.
} 
where $R$ is the internal response matrix of a single super period. $R$ is not necessarily a square matrix. This response matrix is a cyclic matrix of submatrices. It was shown in [19] that the eigenvectors of the response matrix $A$ are

$$
\hat{\mathcal{E}}_{j k}=\frac{1}{\sqrt{N_{s}}}\left(\begin{array}{c}
\hat{E}_{j} \\
\hat{E}_{j} e^{i 2 \pi k / N_{s}} \\
\vdots \\
\hat{E}_{j} e^{i 2 \pi k\left(N_{s}-1\right) / N_{s}}
\end{array}\right)
$$

where $\hat{E}_{j}$ is the $j$-th eigenvector of the matrix $R$. A Discrete Fourier Transform (DFT) can be performed on the eigenvector of Eq. (12) in order to find the spatial spectrum of the eigenvectors. The result is

$$
F_{(j k) l}=\exp \left[i \pi(k+l)\left(1-\frac{1}{N_{s}}\right)\right] \frac{\sin \pi(k+l)}{\sin \frac{\pi}{N_{s}}(k+l)} \sum_{h=0}^{N_{m} / N_{s}-1}\left(\hat{E}_{j}\right)_{h} \exp \left(i \frac{2 \pi}{N_{m}} l h\right)
$$

where $F_{(j k) l}$ is the $l$-th element of the DFT of $\hat{\mathcal{E}}_{j k}$ and $N_{m} / N_{s}$ is the number of BPMs per super period. This results shows that in a case of a perfectly super symmetric ring, the eigenvectors are harmonics of the super periodicity, but instead of being perfect harmonics, their spectrum is now broadened by the spectrum of a single super period. The resulting eigen orbits are convolution between the super-symmetry and a single period. This results in coupled pendulums [19].

\subsection{Broken super-symmetry}

The theoretical analysis of a synchrotron machine with broken super-symmetry is quite complex and is beyond the scope of this paper. Comparison of the eigen orbits of the measured response matrix in the NSLS Xray ring [19] to the eigen orbits of the calculated response matrix, show significant differences. These differences are mainly in the eigen orbits corresponding to the intermediate range of eigenvalues.

\section{Reduction of orbit corrector strengths}

As we mentioned in Sec. 2.2 , that property of the eigen solutions that a small eigenvalue $\lambda_{j}$ corresponds to a small $x_{j}$ orbit change can be used for reducing the corrector strengths without significantly changing the resulting orbit.

Let $\vec{X}_{0}$ be the orbit to be corrected and $\vec{\Theta}$ its corresponding kick vector, calculated by any method (e.g. least square, harmonics, etc). We define the residual orbit to be the difference between the orbit change generated by the vector $\vec{\Theta}$ and the desired orbit change $\vec{X}_{0}$. The corresponding rms average is calculated by

$$
X_{r m s}^{2}=\frac{1}{N_{m}}\left\|A \vec{\Theta}-\vec{X}_{o}\right\|^{2} .
$$

The $\bar{\Theta}$ can be reduced (both, in its rms value and in its maximum component) by subtracting an eigenvector $\hat{\theta}_{j}$ out of it. When the corresponding eigenvalue $\lambda_{j}$ is small, this will result in a small change of the orbit. Thus, a significant decrease of the correctors strength may be achieved without changing much the resulting orbit. We minimize the term

$$
\left\|\vec{\Theta}-r_{j} \hat{\theta}_{j}\right\|^{2}
$$

with respect to $r_{j}$ in order to find the optimal value of $r_{j}$. This results in 


$$
r_{j}=\vec{\Theta} \cdot \hat{\theta}_{j}
$$

In practice, several eigenvectors are used in the optimization of the kick vector. The number $J$ of eigenvectors to reduce the kick vector is determined by the tolerance in the orbit correction. $J$ is the smallest index for which the condition

$$
\sum_{j=1}^{J} r_{j}^{2} \lambda_{j} \geq E
$$

is true, where $E=N_{m} \epsilon^{2}$ and $\epsilon$ is the orbit tolerance. In order to achieve a better optimization, $r_{J}$ can be modified so that the inequality in Eq. (17) becomes equality. The modified $r_{J}$ is

$$
r_{J}^{\text {mod }}= \pm \sqrt{\frac{E-E^{\prime}}{\lambda_{j}}} ; \quad E^{\prime}=\sum_{j=1}^{J-1} r_{j}^{2} \lambda_{j} .
$$

The sign in Eq. (18) is chosen so that the new coefficient $r_{J}^{\text {mod }}$ has the same sign as the old one $r_{J}$. With $J$ found and $r_{J}$ modified, the optimized kick vector is

$$
\vec{\Theta}_{\text {red }}=\vec{\Theta}-\sum_{j=1}^{J} r_{j} \hat{\theta}_{j}
$$

Note, that this process corresponds to orthogonalizing the vector $\vec{\Theta}$ to the eigenvectors $\hat{\theta}_{j}(j=1 />$ $-/>J-1)$.

\section{Orbit correction by decomposition}

In the previous Section, we made use of the eigen solutions to reduce a given kick vector $\vec{\Theta}$, obtained by any method of orbit correction. However, one can directly use an eigenvector decomposition based orbit correction method, thus avoiding the need for reduction. This method will yield the 'minimum' kick vector for a desired accuracy of orbit correction.

\subsection{Global orbit correction}

In order to make the calculations in this section easier, we introduce the 'normalized' eigen kick vectors $\vec{\theta}_{j}=\hat{\theta}_{j} / \sqrt{\lambda_{j}}$ and the corresponding orbit vectors ${ }^{4}$ represent the orbit change corresponding to the $\vec{\theta}_{j}$ normalized eigenvectors and they comprise an orthogonal but not a $\hat{x}_{j}=A \vec{\theta}_{j}$. Comparison of this definition with Eq. (3) and Eq. (4) shows that $\hat{x}_{j}$ is a unit vector. Let $\vec{X}_{o}$ be the orbit to be corrected. The decomposition of it in terms of the $\hat{x}_{j}$ orbits is:

$$
c_{j}=\vec{X}_{o} \cdot \hat{x}_{j}
$$

That is, each $\hat{x}_{j}$ vector is represented in $\vec{X}_{o}$ by the $c_{j}$ coefficient. Let us next define an orbit vector, $\vec{X}_{\epsilon_{0}}$ as:

$$
\vec{X}_{\epsilon_{o}}=\sum_{j=1}^{N_{c}} c_{j} \cdot \hat{x}_{j}
$$

\footnotetext{
They represent the orbit change corresponding to the $\vec{\theta}$, normalized eigenvectors and they comprise an orthogonal but not a complete orthogonal set.
} 
The norm of this vector is $\left\|\vec{X}_{c_{o}}\right\|^{2}=\sum_{j=1}^{N_{c}} c_{j}^{2}$ and $\left\|\vec{X}_{c_{o}}\right\|^{2} \leq\left\|\vec{X}_{o}\right\|^{2}$. Actually, the uncorrectable part of the orbit is ${ }^{5}$ :

$$
\Delta \vec{X}_{r m s}^{2}=\frac{1}{N_{m}}\left(\left\|\vec{X}_{o}\right\|^{2}-\left\|\vec{X}_{c_{o}}\right\|^{2}\right)=\epsilon_{o}^{2}
$$

Substituting Eq. (3) into Eq. (21), we obtain

$$
\vec{X}_{\epsilon_{o}}=A \sum_{j=1}^{N_{c}} c_{j} \vec{\theta}_{j}
$$

that is, the kick vector which corrects the $\vec{X}_{o}$ orbit to $\epsilon_{o}$ accuracy can be obtained from the eigenvector decomposition of this orbit as:

$$
\bar{\Theta}=\sum_{j=1}^{N_{c}} c_{j} \vec{\theta}_{j}=\sum_{j=1}^{N_{c}} c_{j} \hat{\theta}_{j} / \sqrt{\lambda_{j}} .
$$

This method already assures that we are using only the minimum contribution from each eigenvector, thus the kick vector $\vec{\Theta}$ is 'minimized'. However, if one can allow an $\epsilon>\epsilon_{o}$ tolerance in the orbit correction, then some eigenvectors (or part of it) with the smallest eigenvalues can be omitted, further reducing the $\vec{\Theta}$ kick. Actually, we can skip the eigenvectors until the corresponding decomposition coefficients satisfy:

$$
\sum_{j=1}^{J-1} c_{j}^{2} \leq E \quad \text { where } \quad E \equiv N_{m} \epsilon^{2},
$$

where $J$ is the largest index for which Eq. (25) is true. In reality, there is no $J$ for which the equality would be exactly satisfied. Generally, the sum for the first $J-1$ eigenvector is $E^{\prime}<E$ and for the $J$-th eigenvector the sum will be greater then $\mathrm{E}$ :

$$
\sum_{j=1}^{J-1} c_{j}{ }^{2}+c_{J}^{2}=E^{\prime}+c_{J}{ }^{2} .
$$

Therefore, to achieve an $\epsilon$ accuracy and hence better optimization in the orbit correction, we can omit the first $J-1$ eigenvectors and a part of the $J$-th. The modified $J$-th coefficient is

$$
c_{J}^{\text {mod }}=c_{J}-\Delta ; \quad \Delta= \pm \sqrt{E-E^{\prime}} .
$$

The sign of $\Delta$ is chosen to be the same as the sign of $c_{J}$. The resulting optimized kick vector is

$$
\vec{\Theta}_{o p t}=\sum_{j=J}^{N_{c}} c_{j} \vec{\theta}_{j}
$$

\subsection{Including local bumps}

The tolerance on the global RMS orbit, even though very stringent, is usually larger then the tolerance on a few selected PUE's, usually at the ends of insertion devices. In the case of the NSLS X-ray ring, for example, the requirement on the global RMS orbit is $100 \mu$, while before and after the insertion devices it is $20 \mu$. During operation, for each fill of the ring, first the global orbit was

${ }^{5}$ This part of the orbit cannot be corrected by any method with the given set of orbit correctors. 
corrected using harmonic or least-square method then local bumps were implemented to position the orbit more accurately at the insertion devices.

With the decomposition method we successfully implemented the global and local orbit correction at the same time by assigning different weight factors at those PUE's in sensitive positions before and after the insertion devices.

\section{Conclusion}

The methods introduced in this paper are very useful attaining accurate and reliable orbit correction that is beyond the previous methods. These methods also assure that the corrected orbit has the shortest possible trajectory between the PUEs. The reason for some of the eigen orbits being local bumps is still somewhat of a mystery, and needs to be further explored. However, since these "natural" bumps appear in the straight sections where they are usually desired, they facilitate creation of high precision local bumps with minimum disturbance to the rest of the orbit.

\section{References}

[1] A. Jackson, SLAC report No. SRS/NSS/75/103 (1975).

[2] B. Autin and Y. Marti, CERN ISR-MA/73-17 (1973).

[3] A.S. King, M.J. Lee and P. Morton, IEEE Trans. Nucl. Sci., Vol. NS-20, (1973).

[4] E. Close, M. Cornacchia, A.S. King and M.J. Lee, PEP Note-271 (1978).

[5] E. Bozoki, CERN/PS/PSR/85-57 (1985).

[6] T. Risselada, CERN/PS(PSR) 87-90 (1987).

[7] Y. Tang and S. Krinsky, Proc. of the IEEE PAC 1993, Washington DC.

[8] E. Gianfelice and R. Giannini, CERN/PS 85-42 LEA (1985).

[9] R. Raja, A. Russel and C. Ankenbrandt, NIM A242, p. 15-22, (1985).

[10] Y. Cheng and C.S. Hsue, Proc. of the IEEE PAC, V.3, p.1704, (1991).

[11] R. Carr, SLAC/SSRL-0001, (1993).

[12] W.J. Corbett, B. Fong, M. Lee and V. Zieman, SLAC-PUB-6110(A), (1993).

[13] G. Guinard, CERN 77-10 ISRD, 6 June (1977).

[14] W.H. Pres, B.P. Flannery, S.A. Teukolsky and W.T. Vetterling, "Numerical Recepies", Cambridge University Press, Cambridge (1988).

[15] E.D. Courant and H.S. Snyder, Annals of Phys., 3, No. 1, p. 1-48, (1958).

[16] E. Bozoki, Proc. of the Workshop on Modeling Based Accelerator Controls, p. 5, (1987). E. Bozoki, NSLS Tech Notes \#291 and \#296, (1987).

[17] L.H. Yu, E. Bozoki, J. Galayda, S. Krinsky and G. Vignola, NIM A284, pp.268 (1989).

[18] A. Friedman, E. Bozoki, O. Singh and J.D. Smith, Proc. of the IEEE PAC 1993, Washington DC.

[19] A. Friedman and E. Bozoki, To be published in NIM. 